\title{
EFFICIENT CALCULATION OF THE JACOBIAN MATRIX FOR 3D INVERSION OF MT DATA
}

\author{
Karina Palheta Gomes and Marcos Welby Correa Silva
}

\begin{abstract}
The Jacobian or sensitivity matrix is a fundamental component in the implementation of many numerical algorithms for the solution of nonlinear inverse problems. It is also used as a tool for sensitivity analysis and for the assessment of the solutions obtained. However, the computation of the Jacobian by a direct method using finite perturbations of model parameters can be very time consuming and for the 3D case with realistic problem dimensions, it is simply unfeasible. In this work, we present all the steps of a mathematical algorithm based on the adjoint-state method for the assembly of the Jacobian matrix for the 3D magnetotelluric parameter to data mapping obtaining significant reduction in computer execution times. Central to the proposed method is the use of a factorized form of the finite elements matrix for the forward problem. Besides, we solved Maxwell's equations using a finite elements method using tetrahedral shaped elements formulated in terms of the vector magnetic and scalar electric potentials. We assessed the efficiency of the proposed methodology using a numerical model of a simple sedimentary basin. Our approach outperformed a direct, brute-force method in both total execution time and memory usage while maintaining the same level of numerical accuracy. The efficiency of the proposed method makes possible the use of the Jacobian matrix for the inversion of large real 3D MT data sets. Although developed here for the particular case of magnetotellurics the methodology can be extended with reasonable efforts for other similar electromagnetic methods like for example controlled source electromagnetics.
\end{abstract}

Keywords: mathematical models, factored matrix of finite elements, electromagnetism, Efficient calculation of the Jacobian for magnetotelluric data.

RESUMO. A matriz jacobiana ou matriz sensibilidade é uma componente fundamental na implementação de diversos algoritmos numéricos para a solução de problemas inversos não-lineares. Ela também é usada como uma ferramenta na análise de sensibilidade e avaliação dos resultados obtidos. No entanto, o cálculo da matriz jacobiana por um método de diferenças finitas, usando perturbações nos parâmetros de inversão do modelo pode consumir muito tempo e, para o caso 3D com dimensões realistas de problemas geofísicos, é simplesmente inviável. Neste trabalho, apresentamos todas as etapas de um algoritmo matemático baseado no método de estados adjuntos para a montagem da matriz jacobiana para o método magnetotelúrico 3D, obtendo uma redução significativa nos tempos de execução do computador. Central para o método proposto é o uso de uma forma fatorada da matriz de elementos finitos para o problema direto. Para isto, resolvemos as equações de Maxwell usando o método de elementos finitos em malhas 3D tetraedrais, formulados em termos dos potenciais vetorial magnético e escalar elétrico. Nós avaliamos a eficiência da metodologia proposta usando um modelo numérico de uma bacia sedimentar simples. Nossa abordagem se mostrou mais eficiente do que um método direto, de força bruta, tanto em relação ao tempo total de execução quanto ao uso de memória, mantendo o mesmo nível de precisão numérica. A eficiência do método proposto possibilita o uso da matriz jacobiana para a inversão de grandes conjuntos de dados 3D reais de MT. Embora desenvolvida aqui para o caso particular do método magnetotelúrico, a metodologia pode ser estendida para outros métodos eletromagnéticos semelhantes, como por exemplo o método eletromagnético de fonte controlada.

Palavras-chave: modelos matemáticos, matriz de elementos finitos fartorada, eletromagnetismo, Cálculo eficiente da jacobiana para dados magnetotelúricos. 


\section{INTRODUTION}

In the magnetotelluric method of geophysical exploration we use measurements of the natural variation of electromagnetic fields at the surface of the earth or on the sea floor in order to estimate the variations in electrical conductivities of the subsurface. The method works in the frequency domain and has the potential to sample the subsurface from a few hundred meters to hundred of kilometers depending on the period of the measured signals. The inverse problem of magnetotellurics aims to estimate the causative distribution of electrical conductivities from the measured data. The inversion starts with the definition of an interpretive model which is usually created based on the discretization of the target domain into 1D, 2D or 3D regular or irregular, structured or unstructured grids. The 3D case, in particular, puts serious computational challenges on the calculation of model responses, which are obtained numerically, and requires a great deal of computational resources with intense use of CPU time and memory.

This inverse problem is frequently formulated as an unconstrained minimization of a highly nonlinear data misfit, which is solved through linearization and iteration until a specified stopping criteria is achieved. One particular method of this type is the Gauss-Newton algorithm. In this method, the sensitivity matrix $J(p) \in C N \times M$ plays a central role. The elements of $\mathrm{J}$ are the derivatives of data functional with respect to each element in the unknown parameter vector and its assembly is the most time consuming task inside every one inversion iteration (Martins et al. (2010), Ramos et al. (2013), Santos (2013) and Santos et al. (2015)). The size of $\mathrm{J}$ increases as more data are added to the problem and the number of unknowns is increased in order to represent satisfactorily the complexity of the target geological model. Thus, it is of paramount importance to have efficient methods for the computation and storage of this matrix.

The most direct way of computing the elements of $\mathrm{J}$ is calculating the derivatives through finite differences approximation. Here, we refer to this strategy as the brute-force method (Luz, 2012). This procedure of calculating derivatives of an observation set in relation to a given parameter element is done through a small perturbation in the respective parameter and using the ratio of the difference between the observation values to the parameter perturbations. This process must be repeated for each parameter in the model requiring on the order of $\mathrm{M}$ or $2 \mathrm{M}$ solutions of the forward problem if forward or central difference formulas are used respectively. Hence, for 3D problems this approach becomes unfeasible due to the huge number of variables and the cost of the solution of each forward problem.

As an alternative to the brute-force method, many large scale inversion algorithms in geophysics nowadays use a technique known as the adjointstate method that greatly reduces the execution time of the inversion (Rodi (1976), Newman and Alumbaugh (2000), Newman and Boggs (2004), Siripunvaraporn et al. (2005), Lin et al. (2011). In electromagnetic inversion methods, the adjointstate method have been used in Luz (2012) for the solution of the 2D magnetotelluric inverse problem using the Total Variation regularization and in Blanco (2018) for the solution of 3D inverse problem of electroresistivity data using global smooth method with finite elements in a grid of tetrahedral elements.

In this work, we present a detailed mathematical formulation of the Jacobian matrix associated to the 
3D magnetotelluric parameter to data mapping following the steps of Newman and Boggs (2004) and Spitzer (1998). Our approach solves the direct problem using finite elements with tetrahedral elements through a formulation of the vector magnetic and the scalar electric potentials. We solve the finite elements linear system using a direct method based on the software package PARDISO distributed with the Intel Fortran compiler. The data functionals for this problem are the apparent resistivities and phases associated to the impedance tensor; the unknowns are the medium conductivities that are specified at the mesh elements. All these quantities are mathematically defined in the sections below.

This work is organized as follows. Section Forward problem and data functionals describe the basic equations for the definition of the data functionals used in the magnetotelluric inversion problem. Section Derivatives of data functionals develops the expressions for the derivatives of the data functionals with respect to the inversion parameters. Section Computing the Jacobian applies the adjoint-state method to obtain the Jacobian matrix in both model and data space domains. In Results and Discussions we show the results of application of the proposed method for a simulated geological model and finally in Conclusions we wrap up the main points of the work and our perspectives about it.

\section{METHODOLOGY}

\section{Forward problem and data functionals}

The magnetotelluric method is setup in the diffusive regime of the electromagnetic field propagating in a conductive earth. We start with Maxwell's equations for harmonic fields assuming a time dependence of the form eiwt and discarding the displacement current term, then we have

$$
\begin{gathered}
\nabla \times \mathbf{E}=-i \omega \mu_{0} \mathbf{H} \\
\nabla \times \mathbf{H}=\sigma \mathbf{E}
\end{gathered}
$$

The source is assumed to be a plane wave linearly polarized impinging vertically on the earth surface. The modeling domain $\Omega$ is a bounded domain in $\mathrm{R}^{3}$. Homogeneous Dirichlet boundary conditions were properly applied on $\partial \Omega$. We take an approach for the solution of Equations (1) and (2) based on potentials, such that the fields $E$ and $\mathbf{H}$ are given by

$$
\mathbf{H}=\nabla \times \mathbf{A}
$$

$$
\mathbf{E}=-i \omega \mu_{0}(\mathbf{A}+\nabla \psi)
$$

where $\mathbf{A}$ and $\psi$ are the vector and scalar potentials respectively. The components of the electric and magnetic fields, in terms of the potentials, are given by

$$
\begin{array}{ll}
H_{x}^{(1,2)}= & \frac{\partial A_{z}^{(1,2)}}{\partial y}-\frac{\partial A_{y}^{(1,2)}}{\partial z}, \\
H_{y}^{(1,2)}= & \frac{\partial A_{x}^{(1,2)}}{\partial z}-\frac{\partial A_{z}^{(1,2)}}{\partial x}, \\
H_{z}^{(1,2)}= & \frac{\partial A_{y}^{(1,2)}}{\partial x}-\frac{\partial A_{x}^{(1,2)}}{\partial y}, \\
E_{x}^{(1,2)}= & -i \omega \mu_{0}\left(A_{x}^{(1,2)}+\frac{\partial \psi^{(1,2)}}{\partial x}\right), \\
E_{y}^{(1,2)}= & -i \omega \mu_{0}\left(A_{y}^{(1,2)}+\frac{\partial \psi^{(1,2)}}{\partial y}\right), \\
E_{z}^{(1,2)}= & -i \omega \mu_{0}\left(A_{z}^{(1,2)}+\frac{\partial \psi^{(1,2)}}{\partial z}\right),
\end{array}
$$

where index $(1,2)$ is referring to two fields polarizations.

We use the method of nodal finite elements with tetrahedral mesh for the solution of Equations (1) and (2) for the secondary fields (Rijo, 1977). 
Using the above definitions for the field components, we can obtain the characteristic system of linear equations (Jin, 2002):

$$
\mathrm{Su}=\mathbf{h} \text {, }
$$

where the matrix $\mathbf{S}$ is complex and it depends explicitly on the model conductivities $\sigma$ and frequency $\omega, \mathbf{u}$ is the unknown vector holding the potential components

$$
\mathbf{u}=\left[A_{x}, A_{y}, A_{z}, \psi\right]^{T},
$$

and $\mathbf{h}$ is the driving term. In the magnetotelluric method this term is set with the homogeneous Dirichlet's boundary conditions on $\partial \Omega$. All the details of mathematical formulation for Equation (6) concerned to MT method can be found in Vozoff (1991), Berdichevsky and Dmitriev (2002) and Jin (2002).

The magnetotelluric data are defined as the ratio between the horizontal components of the electric and magnetic fields measured at the earth surface (Cagniard, 1953). Expressed by the relation in matrix notation,

$$
\begin{aligned}
{\left[\begin{array}{l}
E_{x} \\
E_{y}
\end{array}\right] } & =\left[\begin{array}{l}
Z_{x x} Z_{x y} \\
Z_{y x} Z_{y y}
\end{array}\right]\left[\begin{array}{l}
H_{x} \\
H_{y}
\end{array}\right] \\
\mathbf{E} & =\mathbf{Z} \mathbf{H},
\end{aligned}
$$

where $Z_{x x}, Z_{x y}, Z_{y x}$ and $Z_{y y}$ are the components of the complex impedance tensor.

The magnetotelluric data functionals, apparent resistivity and phase are given by

$$
\begin{gathered}
\rho_{a(i j)}=\frac{1}{\omega \mu_{0}}\left|Z_{i j}\right|^{2}, \\
\phi_{i j}=\operatorname{atan}^{-1}\left(\frac{\operatorname{Im}\left\{Z_{i j}\right\}}{\operatorname{Re}\left\{Z_{i j}\right\}}\right) .
\end{gathered}
$$

or

$$
\begin{gathered}
\rho_{a}=\left[\begin{array}{ll}
\rho_{x x} & \rho_{x y} \\
\rho_{y x} & \rho_{y y}
\end{array}\right], \\
\phi=\left[\begin{array}{ll}
\phi_{x x} & \phi_{x y} \\
\phi_{y x} & \phi_{y y}
\end{array}\right]
\end{gathered}
$$

\section{Derivatives of data functionals}

In this section we develop the expressions for the derivatives of the data functionals $\rho_{a}$ and $\varphi$ with respect to the unknown conductivities $\sigma$ in the model. We first start writing the derivatives written as function of the impedance $\mathbf{Z}$ and its partial derivative with respect to the unknowns $\sigma$ and then we proceed to write the partial derivatives of $\mathbf{Z}$ in terms of the field potentials $\mathbf{A}$ and $\psi$. We begin with the expression for the complex impedance using Euler's formula,

$$
\mathbf{Z}=|\mathbf{Z}| e^{i \varphi},
$$

and differentiating it with respect to $\rho_{j}$ (the reciprocal of conductivity), we obtain

$$
\frac{\partial \mathbf{Z}}{\partial \rho_{j}}=\frac{|\partial \mathbf{Z}| e^{i \phi}}{\partial \rho_{j}}+i|\mathbf{Z}| e^{i \phi} \frac{\partial \phi}{\partial \rho_{j}}
$$

Using expression $e^{i \phi}=\frac{\mathbf{Z}}{|\mathbf{Z}|}$ (from Equation (13) we have:

$$
\frac{\partial \mathbf{Z}}{\partial \rho_{j}}=\frac{\mathbf{Z}}{|\mathbf{Z}|} \frac{\partial|\mathbf{Z}|}{\partial \rho_{j}}+i \mathbf{Z} \frac{\partial \phi}{\partial \rho_{j}} .
$$

We can identify the real and imaginary parts of this equation, which will be used later as

$$
\frac{1}{|\mathbf{Z}|} \frac{\partial|\mathbf{Z}|}{\partial \rho_{j}}=\operatorname{Re}\left\{\mathbf{Z}^{-1} \frac{\partial \mathbf{Z}}{\partial \rho_{j}}\right\}
$$

$$
\frac{\partial \phi}{\partial \rho_{j}}=\operatorname{Im}\left\{\mathbf{z}^{-1} \frac{\partial \mathbf{Z}}{\partial \rho_{j}}\right\}
$$

Proceeding now with the expression for the apparent resistivity, from Equation (9), we have:

$$
\frac{\partial \rho_{a}}{\partial \rho_{i}}=2 \rho_{a} \frac{1}{|\mathbf{Z}|} \frac{\partial|\mathbf{Z}|}{\partial \rho_{j}} .
$$

Finally using equations 16 and 17 we have 


$$
\begin{gathered}
\frac{\partial \rho_{a}}{\partial \rho_{i}}=2 \rho_{a} \operatorname{Re}\left\{\mathbf{Z}^{-1} \frac{\partial \mathbf{Z}}{\partial \rho_{j}}\right\}, \\
\frac{\partial \phi}{\partial \rho_{i}}=\operatorname{Im}\left\{\mathbf{Z}^{-1} \frac{\partial \mathbf{Z}}{\partial \rho_{j}}\right\} .
\end{gathered}
$$

Similar to Rijo et al. (1977), we used conductivity values in the direct modeling and natural logarithm values of both resistivity and apparent resistivity in the inversion process in order to increase the stability of the inverse problem, hence, we rewrite equations (19) and (20) as:

$$
\begin{gathered}
\frac{\partial \ln \rho_{a}}{\partial \ln \rho_{j}}=-2 \sigma_{j} \operatorname{Re}\left\{\mathbf{z}^{-1} \frac{\partial \mathbf{Z}}{\partial \sigma_{j}}\right\} \\
\frac{\partial \phi}{\partial \ln \rho_{j}}=\operatorname{Im}\left\{\mathbf{z}^{-1} \frac{\partial \mathbf{Z}}{\partial \sigma_{j}}\right\},
\end{gathered}
$$

Differentiating the Equation (8) with respect to $\sigma_{j}$, we have

$$
\frac{\partial \mathbf{E}}{\partial \sigma_{j}}=\frac{\partial \mathbf{Z}}{\partial \sigma_{j}} \mathbf{H}+\mathbf{Z} \frac{\partial \mathbf{H}}{\partial \sigma_{j}}
$$

or in matrix notation:

$$
\begin{aligned}
{\left[\begin{array}{l}
\frac{\partial E_{x}}{\partial \sigma_{j}} \\
\frac{\partial E_{y}}{\partial \sigma_{j}}
\end{array}\right] } & =\left[\begin{array}{ll}
\frac{\partial Z_{x x}}{\partial \sigma_{j}} & \frac{\partial Z_{x y}}{\partial \sigma_{j}} \\
\frac{\partial Z_{y x}}{\partial \sigma_{j}} & \frac{\partial Z_{y y}}{\partial \sigma_{j}}
\end{array}\right]\left[\begin{array}{c}
H_{x} \\
H_{y}
\end{array}\right] \\
& +\left[\begin{array}{ll}
Z_{x x} & Z_{x y} \\
Z_{y x} & Z_{y y}
\end{array}\right]\left[\begin{array}{l}
\frac{\partial H_{x}}{\partial \sigma_{j}} \\
\frac{\partial H_{y}}{\partial \sigma_{j}}
\end{array}\right]
\end{aligned}
$$

The system (24) can be solved using two polarizations of both $\mathbf{E}$ and $\mathbf{H}$ :

$$
\begin{gathered}
\frac{\partial E_{x}^{(1,2)}}{\partial \sigma_{j}}=\frac{\partial Z_{x x}}{\partial \sigma_{j}} H_{x}^{(1,2)}+\frac{\partial Z_{x y}}{\partial \sigma_{j}} H_{y}^{(1,2)} \\
+Z_{x x} \frac{\partial H_{x}^{(1,2)}}{\partial \sigma_{j}}+Z_{x y} \frac{\partial H_{y}^{(1,2)}}{\partial \sigma_{j}} \\
\frac{\partial E_{y}^{(1,2)}}{\partial \sigma_{j}}=\frac{\partial Z_{y x}}{\partial \sigma_{j}} H_{x}^{(1,2)}+\frac{\partial Z_{y y}}{\partial \sigma_{j}} H_{y}^{(1,2)} \\
+Z_{y x} \frac{\partial H_{x}^{(1,2)}}{\partial \sigma_{j}}+Z_{y y} \frac{\partial H_{y}^{(1,2)}}{\partial \sigma_{j}}
\end{gathered}
$$

where index $(1,2)$ is referring to the fields polarizations. Taking the difference between these polarizations in terms of $\frac{1}{H x} \frac{\partial E x}{\partial \sigma_{j}}$ we have:

$$
\begin{gathered}
\frac{1}{H_{x}^{(1)}} \frac{\partial E_{x}^{(1)}}{\partial \sigma_{j}}-\frac{1}{H_{x}^{(2)}} \frac{\partial E_{x}^{(2)}}{\partial \sigma_{j}}=\frac{H_{y}^{(1)}}{H_{x}^{(1)}} \frac{\partial Z_{x y}}{\partial \sigma_{j}}+ \\
\frac{Z_{x x}}{H_{x}^{(1)}} \frac{\partial H_{x}^{(1)}}{\partial \sigma_{j}}+\frac{Z_{x y}}{H_{x}^{(1)}} \frac{\partial H_{y}^{(1)}}{\partial \sigma_{j}}-\frac{\partial Z_{x y}}{\partial \sigma_{j}} \frac{H_{y}^{(2)}}{H_{x}^{(2)}}- \\
\frac{Z_{x x}}{H_{x}^{(2)}} \frac{\partial H_{x}^{(2)}}{\partial \sigma_{j}}-\frac{Z_{x y}}{H_{x}^{(2)}} \frac{\partial H_{y}^{(2)}}{\partial \sigma_{j}} \\
\frac{1}{H_{x}^{(1)}} \frac{\partial E_{x}^{(1)}}{\partial \sigma_{j}}-\frac{1}{H_{x}^{(2)}} \frac{\partial E_{x}^{(2)}}{\partial \sigma_{j}}= \\
\frac{\partial Z_{x y}}{\partial \sigma_{j}}\left(\frac{H_{y}^{(1)}}{H_{x}^{(1)}}-\frac{H_{y}^{(2)}}{H_{x}^{(2)}}\right)+ \\
Z_{x x}\left(\frac{1}{H_{x}^{(1)}} \frac{\partial H_{x}^{(1)}}{\partial \sigma_{j}}-\frac{1}{H_{x}^{(2)}} \frac{\partial H_{x}^{(2)}}{\partial \sigma_{j}}\right)+ \\
Z_{x y}\left(\frac{1}{H_{x}^{(1)}} \frac{\partial H_{y}^{(1)}}{\partial \sigma_{j}}-\frac{1}{H_{x}^{(2)}} \frac{\partial H_{y}^{(2)}}{\partial \sigma_{j}}\right),
\end{gathered}
$$

Similarly, from Equation (26) we rewrote:

$$
\begin{gathered}
\frac{1}{H_{y}^{(1)}} \frac{\partial E_{y}^{(1)}}{\partial \sigma_{j}}-\frac{1}{H_{y}^{(2)}} \frac{\partial E_{y}^{(2)}}{\partial \sigma_{j}}=\frac{\partial Z_{y x}}{\partial \sigma_{j}} \frac{H_{x}^{(1)}}{H_{y}^{(1)}}+ \\
\frac{Z_{y x}}{H_{y}^{(1)}} \frac{\partial H_{x}^{(1)}}{\partial \sigma_{j}}+\frac{Z_{y y}}{H_{y}^{(1)}} \frac{\partial H_{y}^{(1)}}{\partial \sigma_{j}}+\frac{\partial Z_{y x}}{\partial \sigma_{j}} \frac{H_{x}^{(2)}}{H_{y}^{(2)}}- \\
\frac{Z_{y x}}{H_{y}^{(2)}} \frac{\partial H_{x}^{(2)}}{\partial \sigma_{j}}-\frac{Z_{y y}}{H_{y}^{(2)}} \frac{\partial H_{y}^{(2)}}{\partial \sigma_{j}} \\
\frac{1}{H_{y}^{(1)}} \frac{\partial E_{y}^{(1)}}{\partial \sigma_{j}}-\frac{1}{H_{y}^{(2)}} \frac{\partial E_{y}^{(2)}}{\partial \sigma_{j}} \\
=\frac{\partial Z_{y x}}{\partial \sigma_{j}}\left(\frac{H_{x}^{(1)}}{H_{y}^{(1)}}-\frac{H_{x}^{(2)}}{H_{y}^{(2)}}\right)+ \\
Z_{y x}\left(\frac{1}{H_{y}^{(1)}} \frac{\partial H_{x}^{(1)}}{\partial \sigma_{j}}-\frac{1}{H_{y}^{(2)}} \frac{\partial H_{x}^{(2)}}{\partial \sigma_{j}}\right)+ \\
Z_{y y}\left(\frac{1}{H_{y}^{(1)}} \frac{\partial H_{y}^{(1)}}{\partial \sigma_{j}}-\frac{1}{H_{y}^{(2)}} \frac{\partial H_{y}^{(2)}}{\partial \sigma_{j}}\right) .
\end{gathered}
$$


Here, we isolate the derivative in $\frac{\partial Z_{x y}}{\partial \sigma_{j}} \quad$ (27) and we define $K$ as $K=H_{y}^{1} H_{x}^{2}-H_{x}^{1} H_{y}^{2}$

$$
\begin{aligned}
& \frac{\partial Z_{x y}}{\partial \sigma_{j}}=\frac{H_{x}^{(2)}}{K} \frac{\partial E_{x}^{(1)}}{\partial \sigma_{j}}-\frac{H_{x}^{(1)}}{K} \frac{\partial E_{x}^{(2)}}{\partial \sigma_{j}}- \\
& \frac{Z_{x x}}{K}\left(H_{x}^{(2)} \frac{\partial H_{x}^{(1)}}{\partial \sigma_{j}}-H_{x}^{(1)} \frac{\partial H_{x}^{(2)}}{\partial \sigma_{j}}\right)- \\
& \frac{Z_{x y}}{K}\left(H_{x}^{(2)} \frac{\partial H_{y}^{(1)}}{\partial \sigma_{j}}-H_{x}^{(1)} \frac{\partial H_{y}^{(2)}}{\partial \sigma_{j}}\right) .
\end{aligned}
$$

Likewise, we isolate the derivative in $\frac{\partial Z_{y x}}{\partial \sigma_{j}}$

$$
\begin{gathered}
\frac{\partial Z_{y x}}{\partial \sigma_{j}}=-\frac{H_{y}^{(2)}}{K} \frac{\partial E_{y}^{(1)}}{\partial \sigma_{j}}+\frac{H_{y}^{(1)}}{K} \frac{\partial E_{y}^{(2)}}{\partial \sigma_{j}}+ \\
\frac{Z_{y x}}{K}\left(H_{y}^{(2)} \frac{\partial H_{x}^{(1)}}{\partial \sigma_{j}}-H_{y}^{(1)} \frac{\partial H_{x}^{(2)}}{\partial \sigma_{j}}\right)+ \\
\frac{Z_{y y}}{K}\left(H_{y}^{(2)} \frac{\partial H_{y}^{(1)}}{\partial \sigma_{j}}-H_{y}^{(1)} \frac{\partial H_{y}^{(2)}}{\partial \sigma_{j}}\right) .
\end{gathered}
$$

Once we obtained the expression for $Z_{x y}$ and $Z_{y x}$ derivatives we proceeded to the computation of $Z_{x x}$ and $Z_{y y}$ derivatives. Now taking differences of Equations (25) in terms of $\frac{1}{H_{y}} \frac{\partial E_{x}}{\partial \sigma_{j}}$ :

$$
\begin{gathered}
\frac{1}{H_{y}^{(1)}} \frac{\partial E_{x}^{(1)}}{\partial \sigma_{j}}-\frac{1}{H_{y}^{(2)}} \frac{\partial E_{x}^{(2)}}{\partial \sigma_{j}}= \\
\frac{\partial Z_{x x}}{\partial \sigma_{j}}\left(\frac{H_{x}^{(1)}}{H_{y}^{(1)}}-\frac{H_{x}^{(2)}}{H_{y}^{(2)}}\right)+ \\
Z_{x x}\left(\frac{1}{H_{y}^{(1)}} \frac{\partial H_{x}^{(1)}}{\partial \sigma_{j}}-\frac{1}{H_{y}^{(2)}} \frac{\partial H_{x}^{(2)}}{\partial \sigma_{j}}\right)+ \\
Z_{x y}\left(\frac{1}{H_{y}^{(1)}} \frac{\partial H_{y}^{(1)}}{\partial \sigma_{j}}-\frac{1}{H_{y}^{(2)}} \frac{\partial H_{y}^{(2)}}{\partial \sigma_{j}}\right) \\
\frac{1}{H_{x}^{(1)}} \frac{\partial E_{y}^{(1)}}{\partial \sigma_{j}}-\frac{1}{H_{x}^{(2)}} \frac{\partial E_{y}^{(2)}}{\partial \sigma_{j}}= \\
\frac{\partial Z_{y y}}{\partial \sigma_{j}}\left(\frac{H_{y}^{(1)}}{H_{x}^{(1)}}-\frac{H_{y}^{(2)}}{H_{x}^{(2)}}\right)+ \\
Z_{y x}\left(\frac{1}{H_{x}^{(1)}} \frac{\partial H_{x}^{(1)}}{\partial \sigma_{j}}-\frac{1}{H_{x}^{(2)}} \frac{\partial H_{x}^{(2)}}{\partial \sigma_{j}}\right)+ \\
Z_{y y}\left(\frac{1}{H_{x}^{(1)}} \frac{\partial H_{y}^{(1)}}{\partial \sigma_{j}}-\frac{1}{H_{x}^{(2)}} \frac{\partial H_{y}^{(2)}}{\partial \sigma_{j}}\right) .
\end{gathered}
$$

and
Differentiating Equations (5) in relation to $\sigma_{j}$ parameter, we obtain:

$$
\begin{gathered}
\frac{\partial H_{x}^{(1,2)}}{\partial \sigma_{j}}=\frac{\partial}{\partial \sigma_{j}}\left(\frac{\partial A_{z}^{(1,2)}}{\partial y}-\frac{\partial A_{y}^{(1,2)}}{\partial z}\right) \\
=\frac{\partial}{\partial y}\left(\frac{\partial A_{z}^{(1,2)}}{\partial \sigma_{j}}\right)-\frac{\partial}{\partial z}\left(\frac{\partial A_{y}^{(1,2)}}{\partial \sigma_{j}}\right) \\
\frac{\partial H_{y}^{(1,2)}}{\partial \sigma_{j}}=\frac{\partial}{\partial \sigma_{j}}\left(\frac{\partial A_{x}^{(1,2)}}{\partial z}-\frac{\partial A_{z}^{(1,2)}}{\partial x}\right) \\
=\frac{\partial}{\partial z}\left(\frac{\partial A_{x}^{(1,2)}}{\partial \sigma_{j}}\right)-\frac{\partial}{\partial x}\left(\frac{\partial A_{z}^{(1,2)}}{\partial \sigma_{j}}\right) \\
\frac{\partial H_{z}^{(1,2)}}{\partial \sigma_{j}}=\frac{\partial}{\partial \sigma_{j}}\left(\frac{\partial A_{y}^{(1,2)}}{\partial x}-\frac{\partial A_{x}^{(1,2)}}{\partial y}\right) \\
=\frac{\partial}{\partial x}\left(\frac{\partial A_{y}^{(1,2)}}{\partial \sigma_{j}}\right)-\frac{\partial}{\partial y}\left(\frac{\partial A_{x}^{(1,2)}}{\partial \sigma_{j}}\right) \\
-i \omega \mu_{0}\left(\frac{\partial A_{z}^{(1,2)}}{\partial \sigma_{j}}+\frac{\partial}{\partial \sigma_{j}} \frac{\partial \psi^{(1,2)}}{\partial z}\right) \\
\frac{\partial E_{x}^{(1,2)}}{\partial \sigma_{j}}=-i \omega \mu_{0}\left(\frac{\partial A_{z}^{(1,2)}}{\partial \sigma_{j}}+\frac{\partial}{\partial z} \frac{\partial \psi^{(1,2)}}{\partial \sigma_{j}}\right) \\
=-i \omega \mu_{0}\left(\frac{\partial A_{x}^{(1,2)}}{\partial \sigma_{j}}+\frac{\partial}{\partial \sigma_{j}} \frac{\partial \psi^{(1,2)}}{\partial x}\right) \\
=-i \omega \mu_{0}\left(\frac{\partial A_{x}^{(1,2)}}{\partial \sigma_{j}}+\frac{\partial}{\partial x} \frac{\partial \psi^{(1,2)}}{\partial \sigma_{j}}\right) \\
\left.\frac{\partial \sigma_{y}^{(1,2)}}{\partial \sigma_{j}}+\frac{\partial}{\partial y} \frac{\partial \psi^{(1,2)}}{\partial \sigma_{j}}\right)
\end{gathered}
$$

Then, we rewrite Equations (27), (28), (31) and (32) using these potentials and constant $K$. The impedance derivatives are finally expressed as: 


$$
\begin{aligned}
& \frac{\partial Z_{x y}}{\partial \sigma_{j}}=-\frac{i \omega \mu_{0}}{K} H_{x}^{(2)}\left(\frac{\partial A_{x}^{(1)}}{\partial \sigma_{j}}+\frac{\partial}{\partial x} \frac{\partial \psi^{(1)}}{\partial \sigma_{j}}\right)+ \\
& \frac{i \omega \mu_{0}}{K} H_{x}^{(1)}\left(\frac{\partial A_{x}^{(2)}}{\partial \sigma_{j}}+\frac{\partial}{\partial x} \frac{\partial \psi^{(2)}}{\partial \sigma_{j}}\right)- \\
& \frac{Z_{x x}}{K} H_{x}^{(2)}\left[\frac{\partial}{\partial y}\left(\frac{\partial A_{z}^{(1)}}{\partial \sigma_{j}}\right)-\frac{\partial}{\partial z}\left(\frac{\partial A_{y}^{(1)}}{\partial \sigma_{j}}\right)\right]+ \\
& \frac{Z_{x x}}{K} H_{x}^{(1)}\left[\frac{\partial}{\partial y}\left(\frac{\partial A_{z}^{(2)}}{\partial \sigma_{j}}\right)-\frac{\partial}{\partial z}\left(\frac{\partial A_{y}^{(2)}}{\partial \sigma_{j}}\right)\right]- \\
& \frac{Z_{x y}}{K} H_{x}^{(2)}\left[\frac{\partial}{\partial z}\left(\frac{\partial A_{x}^{(1)}}{\partial \sigma_{j}}\right)-\frac{\partial}{\partial x}\left(\frac{\partial A_{z}^{(1)}}{\partial \sigma_{j}}\right)\right]+ \\
& \frac{Z_{x y}}{K} H_{x}^{(1)}\left[\frac{\partial}{\partial z}\left(\frac{\partial A_{x}^{(2)}}{\partial \sigma_{j}}\right)-\frac{\partial}{\partial x}\left(\frac{\partial A_{z}^{(2)}}{\partial \sigma_{j}}\right)\right] \text {, } \\
& \frac{\partial Z_{y x}}{\partial \sigma_{j}}=\frac{i \omega \mu_{0}}{K} H_{y}^{(2)}\left(\frac{\partial A_{y}^{(1)}}{\partial \sigma_{j}}+\frac{\partial}{\partial y} \frac{\partial \psi^{(1)}}{\partial \sigma_{j}}\right)- \\
& \frac{i \omega \mu_{0}}{K} H_{y}^{(1)}\left(\frac{\partial A_{y}^{(2)}}{\partial \sigma_{j}}+\frac{\partial}{\partial y} \frac{\partial \psi^{(2)}}{\partial \sigma_{j}}\right)+ \\
& \frac{Z_{y x}}{K} H_{y}^{(2)}\left[\frac{\partial}{\partial y}\left(\frac{\partial A_{z}^{(1)}}{\partial \sigma_{j}}\right)-\frac{\partial}{\partial z}\left(\frac{\partial A_{y}^{(1)}}{\partial \sigma_{j}}\right)\right]- \\
& \frac{Z_{y x}}{K} H_{y}^{(1)}\left[\frac{\partial}{\partial y}\left(\frac{\partial A_{z}^{(2)}}{\partial \sigma_{j}}\right)-\frac{\partial}{\partial z}\left(\frac{\partial A_{y}^{(2)}}{\partial \sigma_{j}}\right)\right]+ \\
& \frac{Z_{y y}}{K} H_{y}^{(2)}\left[\frac{\partial}{\partial z}\left(\frac{\partial A_{x}^{(1)}}{\partial \sigma_{j}}\right)-\frac{\partial}{\partial x}\left(\frac{\partial A_{z}^{(1)}}{\partial \sigma_{j}}\right)\right]- \\
& \frac{Z_{y y}}{K} H_{y}^{(1)}\left[\frac{\partial}{\partial z}\left(\frac{\partial A_{x}^{(2)}}{\partial \sigma_{j}}\right)-\frac{\partial}{\partial x}\left(\frac{\partial A_{z}^{(2)}}{\partial \sigma_{j}}\right)\right] \text {, } \\
& \frac{\partial Z_{x x}}{\partial \sigma_{j}}=\frac{i \omega \mu_{0}}{K} H_{y}^{(2)}\left(\frac{\partial A_{x}^{(1)}}{\partial \sigma_{j}}+\frac{\partial}{\partial x} \frac{\partial \psi^{(1)}}{\partial \sigma_{j}}\right)- \\
& \frac{i \omega \mu_{0}}{K} H_{y}^{(1)}\left(\frac{\partial A_{x}^{(2)}}{\partial \sigma_{j}}+\frac{\partial}{\partial x} \frac{\partial \psi^{(2)}}{\partial \sigma_{j}}\right)+ \\
& \frac{Z_{x x}}{K} H_{y}^{(2)}\left[\frac{\partial}{\partial y}\left(\frac{\partial A_{z}^{(1)}}{\partial \sigma_{j}}\right)-\frac{\partial}{\partial z}\left(\frac{\partial A_{y}^{(1)}}{\partial \sigma_{j}}\right)\right]- \\
& \frac{Z_{x x}}{K} H_{y}^{(1)}\left[\frac{\partial}{\partial y}\left(\frac{\partial A_{z}^{(2)}}{\partial \sigma_{j}}\right)-\frac{\partial}{\partial z}\left(\frac{\partial A_{y}^{(2)}}{\partial \sigma_{j}}\right)\right]+ \\
& \frac{Z_{x y}}{K} H_{y}^{(2)}\left[\frac{\partial}{\partial z}\left(\frac{\partial A_{x}^{(1)}}{\partial \sigma_{j}}\right)-\frac{\partial}{\partial x}\left(\frac{\partial A_{z}^{(1)}}{\partial \sigma_{j}}\right)\right]- \\
& \frac{Z_{x y}}{K} H_{y}^{(1)}\left[\frac{\partial}{\partial z}\left(\frac{\partial A_{x}^{(2)}}{\partial \sigma_{j}}\right)-\frac{\partial}{\partial x}\left(\frac{\partial A_{z}^{(2)}}{\partial \sigma_{j}}\right)\right] \text {, }
\end{aligned}
$$

and

$$
\begin{gathered}
\frac{\partial Z_{y y}}{\partial \sigma_{j}}=-\frac{i \omega \mu_{0}}{K} H_{x}^{(2)}\left(\frac{\partial A_{y}^{(1)}}{\partial \sigma_{j}}+\frac{\partial}{\partial y} \frac{\partial \psi^{(1)}}{\partial \sigma_{j}}\right)+ \\
\frac{i \omega \mu_{0}}{K} H_{x}^{(1)}\left(\frac{\partial A_{y}^{(2)}}{\partial \sigma_{j}}+\frac{\partial}{\partial y} \frac{\partial \psi^{(2)}}{\partial \sigma_{j}}\right)- \\
\frac{Z_{y x}}{K} H_{x}^{(2)}\left[\frac{\partial}{\partial y}\left(\frac{\partial A_{z}^{(1)}}{\partial \sigma_{j}}\right)-\frac{\partial}{\partial z}\left(\frac{\partial A_{y}^{(1)}}{\partial \sigma_{j}}\right)\right]+ \\
\frac{Z_{y x}}{K} H_{x}^{(1)}\left[\frac{\partial}{\partial y}\left(\frac{\partial A_{z}^{(2)}}{\partial \sigma_{j}}\right)-\frac{\partial}{\partial z}\left(\frac{\partial A_{y}^{(2)}}{\partial \sigma_{j}}\right)\right]- \\
\frac{Z_{y y}}{K} H_{x}^{(2)}\left[\frac{\partial}{\partial z}\left(\frac{\partial A_{x}^{(1)}}{\partial \sigma_{j}}\right)-\frac{\partial}{\partial x}\left(\frac{\partial A_{z}^{(1)}}{\partial \sigma_{j}}\right)\right]+ \\
\frac{Z_{y y}}{K} H_{x}^{(1)}\left[\frac{\partial}{\partial z}\left(\frac{\partial A_{x}^{(2)}}{\partial \sigma_{j}}\right)-\frac{\partial}{\partial x}\left(\frac{\partial A_{z}^{(2)}}{\partial \sigma_{j}}\right)\right] .
\end{gathered}
$$

\section{Computing the Jacobian}

Once we knew how to evaluate the impedance derivatives $\frac{\partial Z_{x x}}{\partial \sigma_{j}}, \frac{\partial Z_{x y}}{\partial \sigma_{j}}, \frac{\partial Z_{y x}}{\partial \sigma_{j}}$ and $\frac{\partial Z_{y y}}{\partial \sigma_{j}}$ we used their expressions in Equations (21) and (22), to finally obtain the problem Jacobian.

Derivatives of the fields' potentials with respect to $\sigma_{j}$ are performed by differentiation of the global finite element system matrix:

$$
\text { Su }=\mathbf{h} \text {. }
$$

In this system, $\mathbf{S}$ is the global, sparse, symmetric and positive definite matrix, $\mathbf{u}$ represents the solution vector whose components are the scalar potential $\psi$ and the 3 components of the vector potential $\mathbf{A}$ in all grid nodes and $\mathbf{h}$ is the problem source vector.

Deriving the matrices from Equation (43) for the secondary potentials, we have: 


$$
\frac{\partial \mathbf{u}}{\partial \sigma_{j}}=\mathbf{S}^{-1}\left(\frac{\partial \mathbf{h}}{\partial \sigma_{j}}-\frac{\partial \mathbf{S}}{\partial \sigma_{j}} \mathbf{u}\right)
$$

Equation (44) provides the sensitivity of all secondary potentials components in all grid nodes. After composing the vector within parenthesis, the global matrix derivatives and the source vector are analytically calculated and implemented including the subtraction and the matrix product using the same steps employed for the elementary matrices assembly.

As the derivatives related to the sensitivity calculation in relation to $\sigma_{j}$ are equal to zero in all nodes except in the ones that are part of the inversion cell $j$, the number of operations necessary for this vector computation is small compared to the same operations for the computation of the data and the total time used in this process is reduced to a small fraction of time relative to the matrix $\mathbf{S}$ factorization and for computation of the systems solutions.

Therefore, calculating the sensitivity of the potentials components, for each frequency, may be performed by only factoring the coefficient matrix $\mathbf{S}$ once along with performing a number of backward substitutions equal to the number of parameters. To each new frequency, a new matrix $\mathbf{S}$ is generated and a new factorization together with new backward substitutions are necessary. However, in underdetermined problems, in which the number of parameters in greater than the number of observations, we can apply a simple procedure that reduces the number of necessary backward substitutions. For the most simple case in which we are interested only in the sensitivity of one of the potentials components at only one measurement position whose measurement is associated to only one node in the grid, we could select the desired sensitivity $J_{i j}$ simply through premultiplying Equation (44) by a row matrix of $N$ elements whose values are 1 in the position corresponding to the node for which we want to extract the derivative and 0 for all the others. We represent this row as a transposed matrix $\mathbf{Q}$, here called selection matrix:

$$
J_{i j}=\mathbf{Q}^{T} \frac{\partial \mathbf{u}}{\partial \sigma_{j}}=\mathbf{Q}^{T} \mathbf{S}^{-1}\left(\frac{\partial \mathbf{h}}{\partial \sigma_{j}}-\frac{\partial \mathbf{S}}{\partial \sigma_{j}} \mathbf{u}\right),
$$

If instead of using expression 45 we calculated the value of the sensitivity $\mathrm{Jij}$ by transposing this equation, utilizing the symmetry of matrix $S$, the required number of solutions for the equation system is reduced to one:

$$
J_{i j}=\left[\mathbf{Q}^{T} \frac{\partial \mathbf{u}}{\partial \sigma_{j}}\right]^{T}=\left(\frac{\partial \mathbf{h}}{\partial \sigma_{j}}-\frac{\partial \mathbf{S}}{\partial \sigma_{j}} \mathbf{u}\right)^{T} \mathbf{S}^{-1} \mathbf{Q},
$$

The solution of the system $\mathbf{S}^{-1} \mathbf{Q}$ is calculated and the product by the vector within parenthesis involves a small number of operations since a few elements of this vector are not null. For $N$ measurement positions, we generate a column vector $\mathbf{J}_{j}$ of $8 \mathrm{~N}$ observations because the MT data are composed of phase and amplitude of 4 impedance components performing $N$ backward substitutions that generate the columns of the sensitivity matrix $\mathbf{J}$ that correspond to the $\sigma_{j}$ parameter when computed for all frequencies.

\section{RESULTS AND DISCUSSIONS}

For comparison, the calculation of the 3D MT sensitivity matrix approximation using both the adjoint-state method and brute-force method was applied to a geologic model of a 3D sedimentary basin similar to what is proposed by Gaino (2012) as shown in Figure 1. In order to better evaluate the adjoint-state and brute-force methods, we 
attributed to this model structural background 4 rectangular blocks, marked by letters $\mathrm{A}, \mathrm{B}, \mathrm{C}$, and $D$ with tops respectively placed at $2.5 \mathrm{~km}, 2 \mathrm{~km}$, $1.5 \mathrm{~km}$ and $1 \mathrm{~km}$, and a basement at a depth of 3 $\mathrm{km}$. We included these blocks intending to simulate geoelectric structures with abrupt discontinuities similar to structures found in intracratonic basins. To simulate this model, we attributed resistivity values of $50 \Omega \mathrm{m}$ (blue region) and $1600 \Omega \mathrm{m}$ (red region) to the sedimentary package and the basin basement, respectively, as illıstrated in Finu ure ?

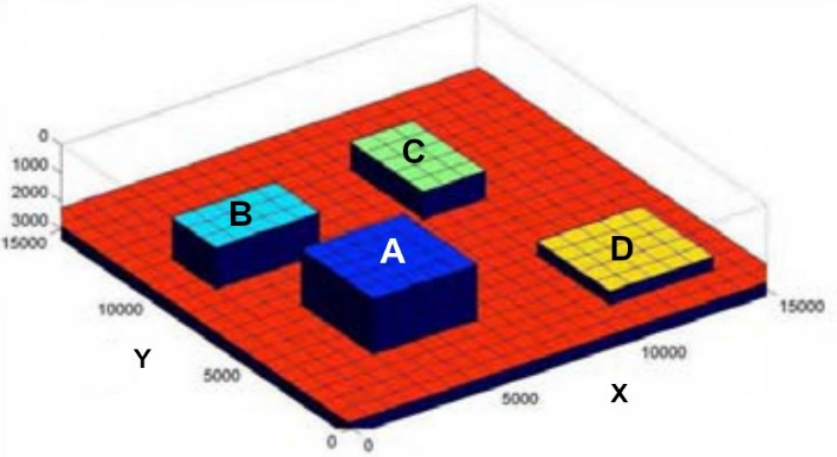

Figure 1 - Structural background synthetic model as proposed by Gaino (2012).

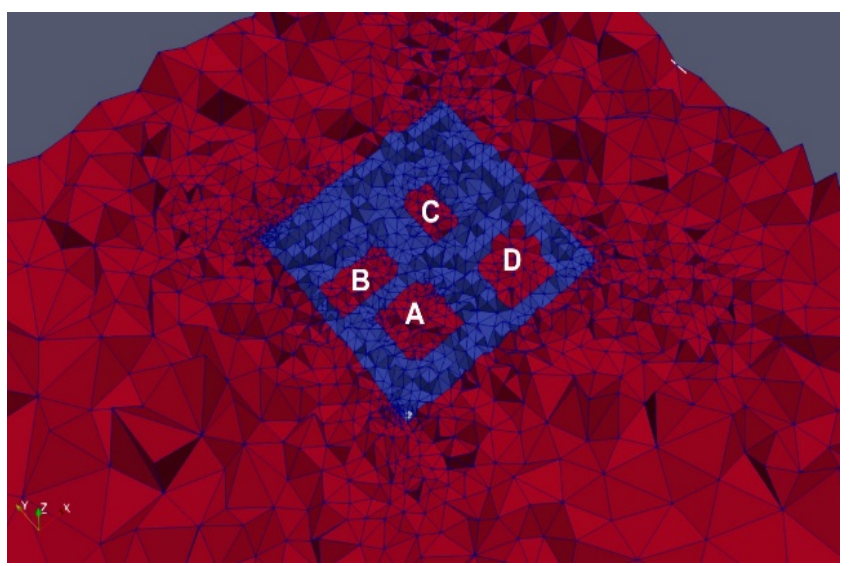

Figure 2 - 3D unstructured finite elements mesh for the model of Gaino (2012).

For generating the magnetotelluric observations we simulated Profiles A and B. Profile A intercepts blocks $A$ and $B$ at $y=4 \mathrm{~km}$ while Profile $B$ intercepts blocks $C$ and $D$ at $y=11 \mathrm{~km}$. The sensors are distributed in 19 measurement points in each profile and are equally spaced by $1 \mathrm{~km}$, as illustrated in Figure 3 over the finite elements grid.

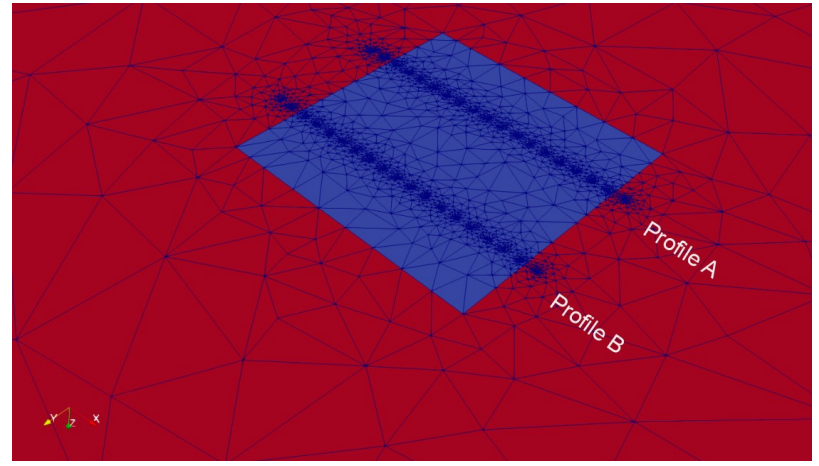

Figure 3 - Magnetotelluric measurements distributed along Profiles $A$ and $B$ sectioning the heterogeneity represented in the 3D finite elements grid.

Figures 4 and 5 are displaying the sensitivity matrix components from the magnetotelluric data calculated by both the adjoint-state (left side) and brute-force (right side) methods. These results are shown as pseudosections of $\rho_{x y}$ and $\rho_{y x}$ for Profiles $A$ and $B$. The agreement between the results from both methods is significant for any frequency and any sounding position. It is important to mention that for the presented model we have only one paramater to be estimated. Figures 6 and 7 show the sensitivity matrix components for the MT method obtained by both the adjoint-state (left side) and brute-force (right side) methods through pseudosections of phases $\varphi_{y x}$ and $\varphi_{y x}$ from Profiles $A$ and B. Similar to what occurs for resistivity, the adjoint-state method presents excellent accuracy compared to the results obtained through the brute-force method for both profiles.

It is important to state that both methods used here are calculated using derivatives obtained through numerical techniques, hence, both methods present an inherent error due to floating point operations. We can call the adjoint-state method 
semi-analytical because we solve the forward problem numerically but regarding the computation of the derivatives, the method is exact. We can say that the accuracy in the computation of the Jacobian by our method is limited by the numerical accuracy of the solution of the underlying linear system and the floating point precision used. The tests were performed in a notebook with a Core $17-7700 \mathrm{HQ}$ $3.6 \mathrm{GHz}$ processor having a total of $16 \mathrm{~GB}$ of RAM memory. The model displayed in Figure 1 was discretized with a finite element mesh of approximately 129,000 nodes which means that we had around 516,000 variables once we consider the four potential components in each node. All the code used was implemented in the Fortran programming language with the Intel Ifort compiler suite. To solve the linear system we used the PARDISO package from Intel MKL library. Although the dimensions of the numerical model in these tests are still small compared to that of real problems it already shows the potential for saving in execution time due to our methodology. To calculate 3D MT data in only one frequency and 38 sounding positions, it was necessary 4.5 minutes and around 9.2 GB of RAM memory. By the brute-force method, the Jacobian calculation took 9.0 minutes due to the cost of two solutions of direct problem to obtain the derivatives by finite differences. For adjoint-state method, the same calculation took 8.7 minutes because this technique uses the solution of only one direct problem (with one full factorization) and 38 backward substitutions (one for each measurement position).

Initially, one can state there is no great advantage on using the proposed methodology. However, here we calculated the derivative in relation to only one parameter. For a problem of 1000 parameters, for example, the brute-force method would demand
2,000 solutions of the direct problem while the adjoint-state method would demand the same number of solutions presented here along with 38 backward substitutions! Only the vectors with the parameters would be greater and the matrix derivative computation of Equation (46) would take longer but these steps correspond to compose the least fraction of the processing time, less than $1 \%$. Using the same finite elements grid, the brute-force method would take 150 hours to calculate the Jacobian while the adjoint-state method would take less than 15 minutes.

\section{CONCLUSIONS}

Sensitivity analysis is a very important component in the overall task of inversion of geophysical data. It makes possible to recognize how the many different inversion parameters are constrained by the data. This analysis is effected with the use of the Jacobian matrix of the parameter to data mapping corresponding to the direct problem. The use of the Jacobian matrix also makes possible the use Gauss-Newton type nonlinear minimization algorithms that have better convergence properties resulting in a reduced number of iterations and less dependence on the initial approximations.

In this work, we demonstrate a mathematical formulation for the Jacobian computation of magnetotelluric method. We obtained a solution from the linear system related to the direct MT problem and emphatize the advantages of the implemented solution. In our approach, the observed data are apparent resistivities and phases related to the impedance tensor and to the parameters that are the conductivities of the geoelectric model. 

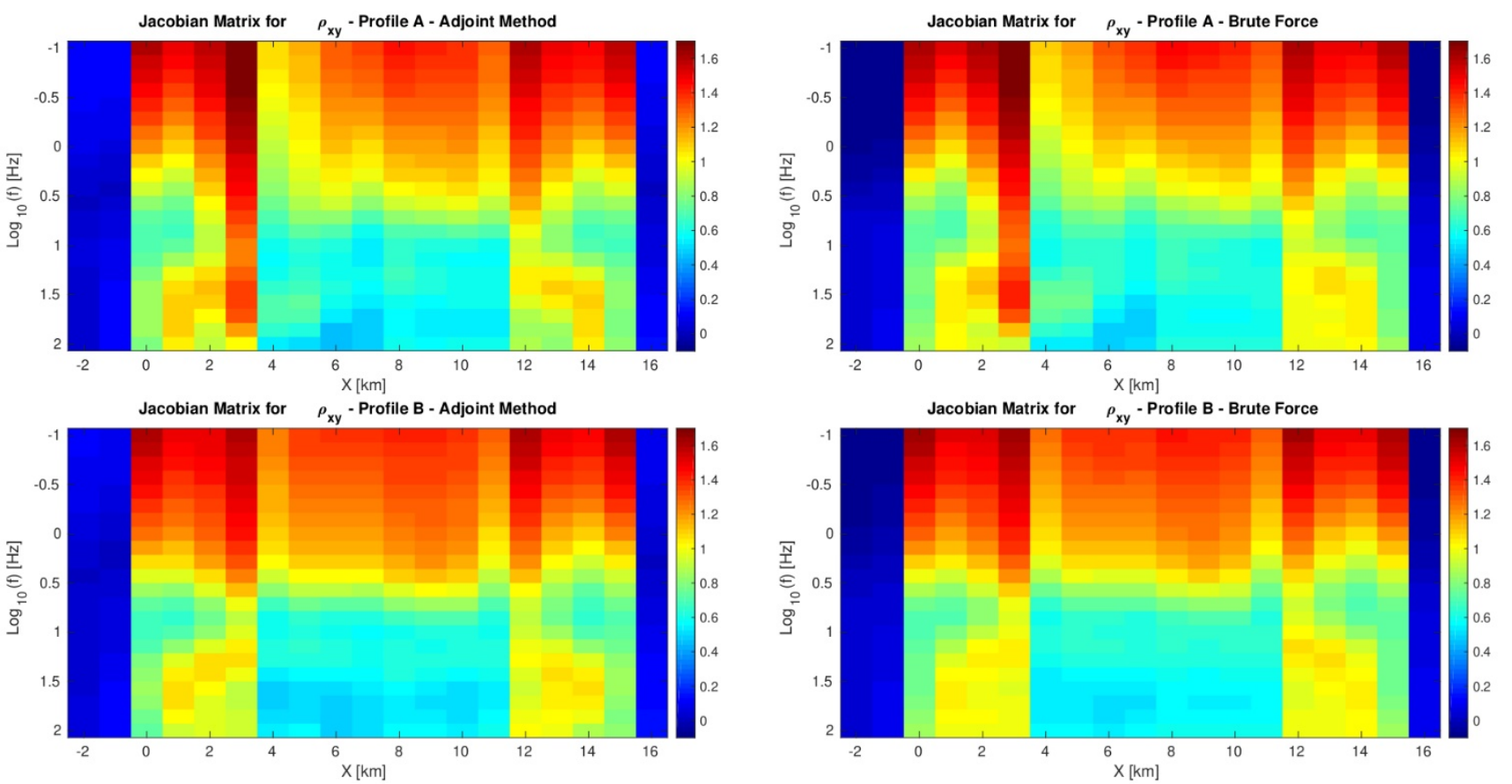

Figure 4 - pxy derivative in Profiles A and B: adjoint vs. perturbation.
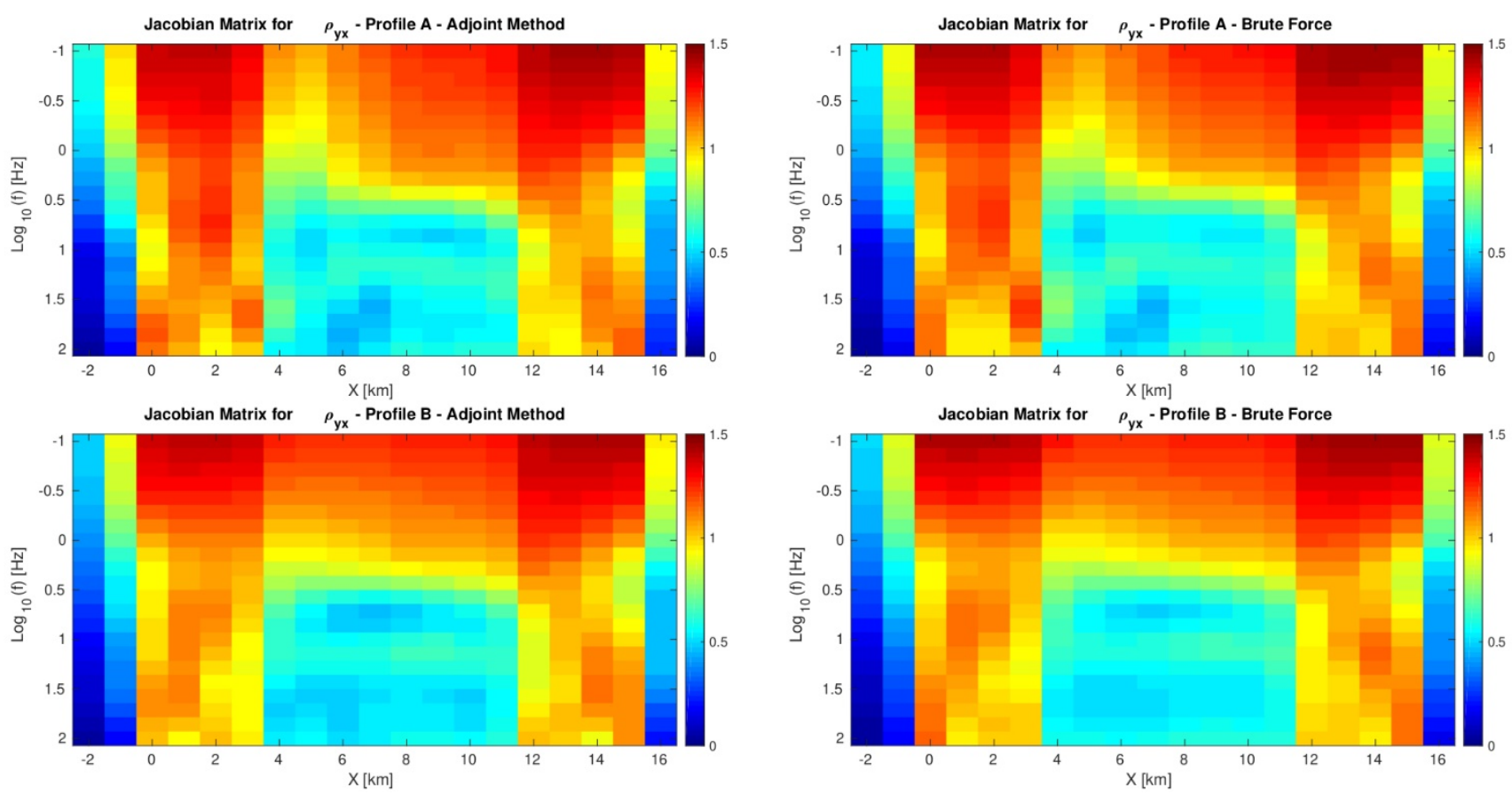

Figure 5 - pyx derivative in Profiles A and B: adjoint vs. perturbation. 

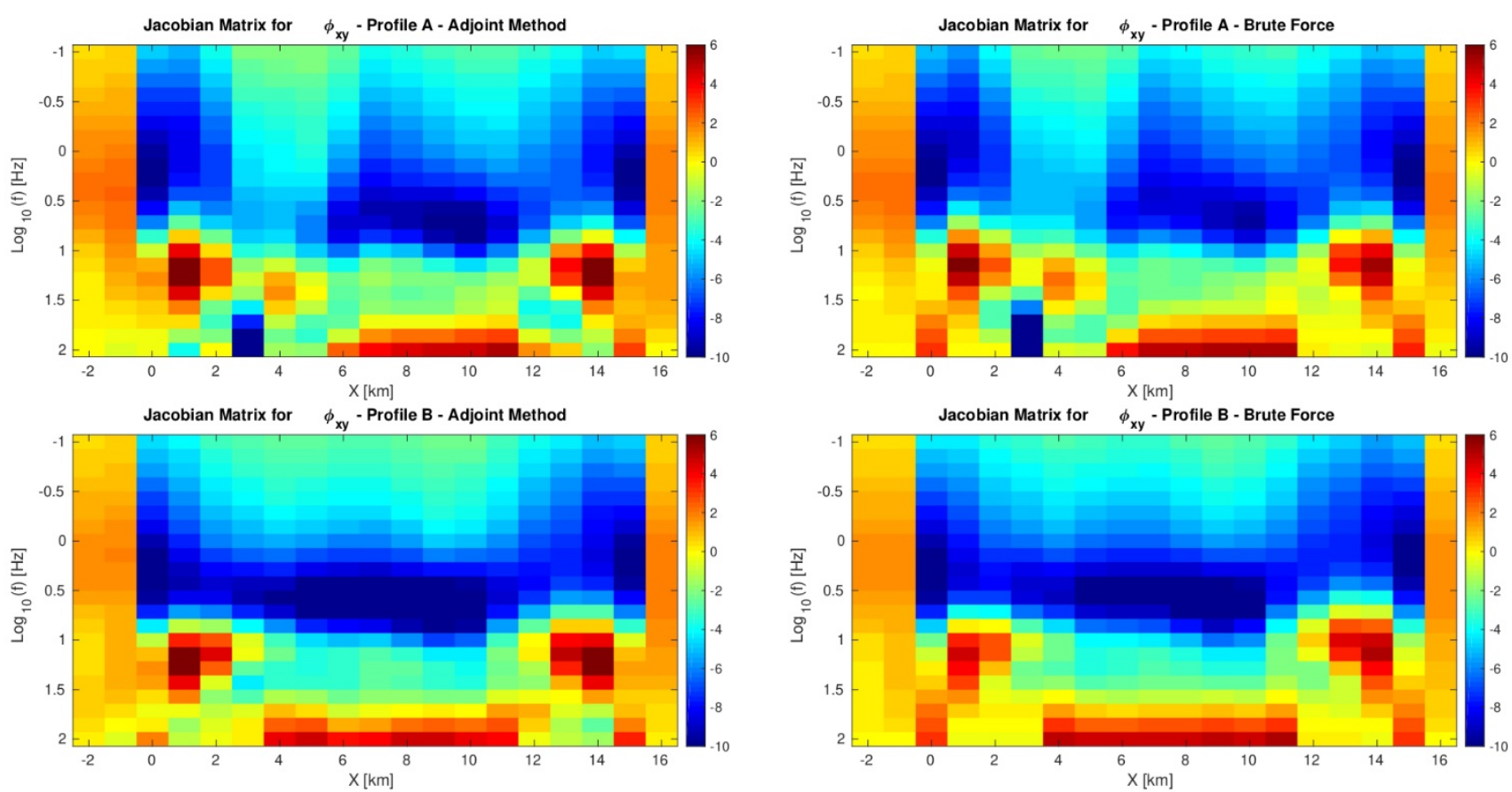

Figure $6-\varphi_{x y}$ derivative in Profiles A and B: adjoint against perturbation.
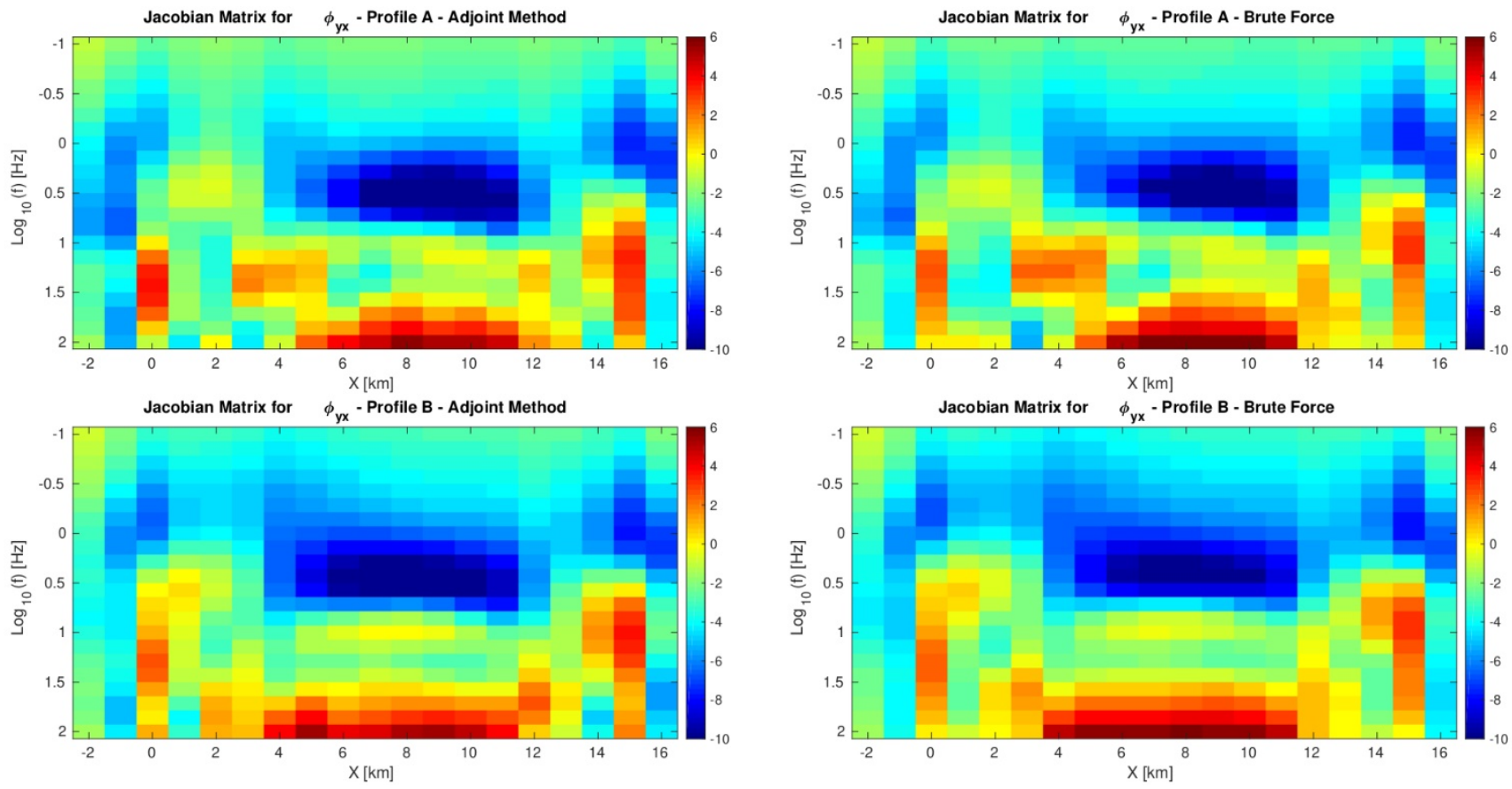

Figure 7 - $\varphi y x$ derivative in Profiles A and B: adjoint against perturbation. 
The 3D direct problem was solved using the finite elements method using tetrahedral elements. We applied first the brute-force method to compute the Jacobian matrix elements. Next, we applied the proposed methodology using the adjoint-state method obtaining huge reductions in the total computational time.

We applied the proposed method to a simple model of a sedimentary basin. Although a simplified version of a true geological situation, the numerical tests corroborate the effectiveness of our approach when compared to the direct approach of the bruteforce method. Developed here for the particular case of inversion of magnetotelluric data, our approach can easily be adapted to other electromagnetic inverse problems.

\section{ACKNOWLEDGEMENTS}

The authors would like to thank the Geophysics Postgraduate Program of the Universidade Federal do Pará (UFPA) for providing the opportunity to develop this research. The CNPq for the financial support and finally - but not less important - the authors are indebted to UFPA for hosting our research team and by the variety of knowledge available throughout this institution.

\section{REFERENCES}

BERDICHEVSKY, MARK N. \& DMITRIEV, V. I., 2002, Magnetotellurics in the context of the theory of ill-posed problems: Society of Exploration Geophysicists. 230 pp.

BLANCO, F. R., 2018, Inversão 3D de dados de eletrorresistividade usando o arranjo dipolodipolo: Master's dissertation, Universidade Federal do Pará. Brazil. 75 pp.
CAGNIARD, L., 1953, Basic theory of the magnetotelluric method of geophysical prospecting: Geophysics, 18, 605-635.

GAINO, M. B., 2012, Estimativa da profundidade do embasamento na Bacia Potiguar usando inversão gravimétrica: Master's dissertation, Universidade Federal do Rio Grande do Norte. Brazil. 175 pp.

Jin, J., 2002, The finite element method in electromagnetics - 2nd ed.: John Wiley and Sons. $753 \mathrm{pp}$.

Lin, C. H., H. D. Tan, and T. Tong, 2011, Three-dimensional conjugate gradient inversion of magnetotelluric full information data: Applied Geophysics, 8, 1-10.

Luz, E. d. C., 2012, Inversão de dados eletromagnéticos com o regularizador variação total e o uso da matriz de sensibilidade aproximada: Master's dissertation, Universidade Federal do Pará. Brazil. 58 pp.

MARTINS, C. M., V. C. BARBOSA, AND J. B. SILVA, 2010, Simultaneous 3D depth-tobasement and density-contrast estimates using gravity data and depth control at few points: Geophysics, 75, 121-I28.

NEWMAN, G. A., AND D.L. ALUMBAUGH, 2000, Three-dimensional magnetotelluric inversion using non-linear conjugate gradients: Geophysical Journal International, 140, 410- 424.

NEWMAN, G. A., AND P. T. BOGGS, 2004, Solution accelerators for large-scale threedimensional electromagnetic inverse problems: Inverse Problems, 20, S151-S170.

RAMOS, L.C., MARTINS C \& SILVA J. 2013, Procedimentos lineares de inversão gravimétrica para estimar o relevo do embasamento de bacias sedimentares. In: 13th International Congress of the Brazilian Geophysical Society \& EXPOGEF, Rio de Janeiro, Brazil, Society of Exploration 
Geophysicists and Brazilian Geophysical Society, 531-536.

RIJO, L., 1977, Modeling of electric and electromagnetic data: $\mathrm{PhD}$ thesis, University of Utah. 138 pp.

RIJO, L., W. PELTON, E. FEITOSA, AND S. WARD, 1977, Interpretation apparent from Apodi Valley, Rio Grande do Norte, Brazil: Geophysics, 42, 811- 822.

RODI, W. L., 1976, A Technique for Improving the Accuracy of Finite Element Solutions for Magnetotelluric Data: Geophysical Journal International, 44, 483-506.

SANTOS, R D. C. S., 2013, Inversão gravimétrica rápida do relevo do embasamento aplicando o funcional da variação total: Master's dissertation, Universidade Federal do Pará. Brazil. 51 pp.
SANTOS, D. F., J. B. SILVA, C. M. MARTINS, R. D. C. S. DOS SANTOS, L. C. RAMOS, AND A. C. M. DE ARAÚJO, 2015, Efficient gravity inversion of discontinuous basement relief: Geophysics, 80, G95-G106.

SIRIPUNVARAPORN, W., G. EGBERT, Y. LENBURY, AND M. UYESHIMA, 2005, Threedimensional magnetotelluric inversion: dataspace method: Physics of the Earth and Planetary Interiors, 150, 3-14. (Electromagnetic Induction in the Earth).

SPITZER, K., 1998, The three-dimensional DC sensitivity for surface and subsurface sources: Geophysical Journal International, 134, 736-746.

VOZOFF K. 1991. The magnetotelluric method. In: Electromagnetic Methods in Applied Geophysics: Volume 2, Application, Parts A and B: Society of Exploration Geophysicists, p. 641-712. 